\title{
Creencias y representaciones de los profesores de lenguas extranjeras sobre la influencia de los factores motivacionales y emocionales en los alumnos y en las alumnas*
}

\author{
NiEves RodríGueZ-PÉREZ \\ Universidad de Oviedo
}

Recibido: 20 mayo 2012 / Aceptado: 17 marzo 2013

ISSN: $1697-7467$

\begin{abstract}
In the classroom context we find students with active implication on learning a language for whom the acquisition of another language-culture may be a gratifying experience. On the contrary, others show disinterest and difficulties. The enthusiasm of some and the disenchantment of others is usually explained as the motivational-emotional factors generated by learning a new language. Our question is whether such factors also influence the two student-groups in a different way. The aim of this study is to bring forward the opinions FL teachers have. The results shown belong to a survey performed on FL teachers who belong to different educational stages.
\end{abstract}

Keywords: motivation, gender, individual differences, attitudes

Kindergarten and Primary Teachers' Perceptions $f$ the Use of ICT in the English Teaching and Learning Process: A Case Study

RESUMEN: En el contexto del aula encontramos alumnos que se implican activamente en el aprendizaje de idiomas, para los cuales aprender otra lengua-cultura resulta gratificante. Sin embargo, otros muestran desinterés y dificultad. El entusiasmo de unos o el desencanto de otros suele explicarse como el efecto de los factores motivacionales-afectivos que genera en el aprendiz la adquisición de otro idioma. Nos preguntamos, además, si estos factores influyen de manera diferente en los alumnos que en las alumnas. El objetivo de este estudio es conocer las opiniones de los docentes. Presentamos los resultados de una encuesta realizada por profesores de idiomas de tres etapas educativas.

Palabras clave: motivación, género, diferencias individuales, actitudes.

\section{INTRODUCCIÓN}

Las diferentes teorías de la motivación en el aprendizaje (Alonso Tapia, 2005; Muñoz, 2002) coinciden en afirmar la existencia de diferentes variables (expectativas, autoestima, metas, atribuciones, reforzadores, emociones,...) que influyen de manera positiva o negativa

"Esta investigación se enmarca en el contexto de una tesis doctoral que lleva por título Motivación y emoción en el proceso de enseñanza-aprendizaje de idiomas. El pensamiento del profesor, fundamentada en una encuesta realizada a docentes de diferentes ámbitos educativos. 
en el proceso de enseñanza-aprendizaje en general. Sin embargo, la adquisición de un idioma posee características especiales (Pavlenko, 2002; Arnold, 2000). Si consideramos que el lenguaje es un canal de comunicación social, un sistema de códigos unidos a la identidad del sujeto, la adquisición de una nueva lengua puede producir efectos sobre la personalidad del aprendiz (Dörnyei, 2009; Wazel, 2001; Pavlenko, 2002). Al incorporarse elementos nuevos de la otra realidad cultural algunos investigadores apuntan al desarrollo de una nueva identidad, el alumno no solo ha de adquirir información nueva sino también elementos simbólicos de la otra comunidad etnolingüística. En otras palabras, el aprendizaje de un idioma implica "una alteración de la autoimagen, la adopción de nuevas conductas sociales y culturales y de nuevas formas de ser" (Williams y Burden, 1999: 123).

Diversos estudios sobre la adquisición de idiomas señalan la emoción como un componente esencial de la actividad cognitiva y subrayan que emoción y cognición no se pueden separar (Arnold, 2000; LeDoux, 1999). Los enfoques para abordar la multiplicidad de la dimensión afectiva son muy diversos, en ella influyen factores personales (variables como la edad, el género, las experiencia anteriores,...) o diferencias individuales (cognitivas, afectivas y de personalidad). Unos autores destacan, entre las múltiples variables afectivas, la actitud y la motivación que los alumnos muestran hacia el conocimiento de la nueva lengua-cultura y hacia sus hablantes (Gardner y Lambert, 1972; Spolsky, 1989; Dörnyei, 2009), otros enfatizan la importancia en el proceso de aprendizaje de los estados emocionales del alumno, los diferentes aspectos de su personalidad — confianza, inhibición, autoestima, ansiedad,...- o la influencia de los factores socioculturales - la relación del alumno con los hablantes de la otra cultura- (Oxford y Shearin, 1994). En nuestra investigación nos centraremos en aquellos factores individuales — la ansiedad, la empatía, la actitud- y en aquellos rasgos de la personalidad del alumno - introversión y extraversión- que tienen un gran alcance en el estudio del idioma.

De acuerdo con Rubio Alcalá (2004) el aprendizaje de un idioma es un proceso lento que impide que el profesor y el aprendiz obtengan resultados inmediatos, circunstancia que puede resultar frustrante. En el aula de idiomas el profesor, los alumnos y la situación grupal están sometidos a interacciones de tipo psíquico que crean una atmósfera específica. El profesor se encuentra influenciado por los alumnos y éstos por el inconsciente del enseñante, por su actitud o su lenguaje corporal - gestos, miradas, expresiones faciales-. Además, en este contexto las situaciones en la clase de idiomas pueden ser, y con frecuencia lo son, muy variadas e informales pues a menudo los docentes incorporan actividades que en ocasiones comprometen emocionalmente a los participantes y que pueden generar sentimientos de pérdida de control, incompetencia o pasión — canciones, juegos que invitan a expresar opiniones y emociones,...- . Si a estas circunstancias añadimos los afectos positivos y/o negativos que genera la aprehensión de una nueva lengua-cultura nos encontramos ante una situación didáctica cargada de sentimientos positivos o negativos que el docente ha de saber reconocer y gestionar de manera adecuada, puesto que ello afecta al propio alumno y a su forma de dar la clase.

En los planteamientos actuales de enseñanza se opta por un enfoque comunicativo en el que priman las interacciones orales en la lengua meta, un vehículo lingüístico que el aprendiz no controla y que, en consecuencia, le obliga a esforzase y arriesgarse estando expuesto a cometer errores. Sentimientos como la ansiedad parecen ser fruto de las interacciones en el uso de la lengua extranjera, lo cual justifica que "aprender un idioma genera más ansie- 
dad que aprender cualquiera otra asignatura" (Rubio Alcalá 2004: 17). La relevancia de la influencia de la ansiedad en el aprendizaje de una lengua extranjera ha sido demostrada en investigaciones llevadas a cabo desde el Enfoque Humanístico y desde diversos paradigmas como el psicolingüístico y el sociolingüístico.

Aunque aún falta mucho por investigar sobre la relación entre ansiedad y género, algunos autores como Campbell (1999) manifiestan que ésta afecta de forma diferente a las mujeres que a los varones. En su opinión las alumnas experimentan mayor ansiedad que los alumnos. Quizás se deba a que las alumnas tienden a experimentar mayor miedo al fracaso (Miller, Finley y Mckinley, 1990). Campbell y Shaw (1994) comprobaron que existía diferencia de género en relación al número de horas de clase. A comienzos del año escolar apenas existía diferencia de género pero a finales del curso los varones presentaban menor ansiedad que las mujeres.

Otro rasgo de la personalidad de los alumnos que influye en la adquisición de un idioma es su carácter, extravertido o introvertido. Por definición los alumnos extravertidos son aquellos que participan en diálogos, les gusta expresar su opinión, hablar, se comunican con facilidad, aprenden mejor y más rápido un idioma, son sociables, abiertos a nuevas impresiones, expresan lo que sienten, lo cual favorece el aprendizaje (Bernaus, 2001). Por el contrario, los introvertidos conservan su energía y en ocasiones pierden la excitación de encontrarse con nuevas personas y de experimentar situaciones diferentes. Bernaus (2001: 80) asegura que los alumnos extravertidos consiguen mejores resultados en las pruebas orales y los introvertidos en lectura y escritura, afirmación que debe inducir al docente a reflexionar sobre las estrategias de enseñanza. Es comúnmente aceptado que las alumnas poseen un carácter más extravertido que los alumnos, no obstante autores como Ray Crozier (2001) no han encontrado relación entre estas características de la personalidad y el género. De sus investigaciones se deriva que en Primaria los niños extravertidos obtienen mejores rendimientos, mientras que en Secundaria y Universidad los introvertidos alcanzan resultados superiores. Otras investigaciones sugieren que los varones se muestran más extravertidos, impulsivos y obtienen mejores rendimientos en tareas numéricas, mientras que las mujeres se muestran más extravertidas y el rendimiento es más alto en tareas verbales (Eysenck y Wilson, 1981).

Generalmente se acepta que el carácter extravertido genera estímulos emocionalmente positivos, entre otros motivos porque se favorece la interacción y, por ello, un mayor input y output (Krashen, 1985). Entwistle y Wilson (1977) reconocen en los introvertidos mayor capacidad para obtener éxito académico, superior al de los extravertidos, porque aunque son menos habladores, saben escuchar, piensan y aprenden igual o más que los anteriores.

Guiora et al. (1972) ponen de manifiesto una correlación positiva entre empatía y competencia en la lengua. Es comprensible que si, como mantiene Goleman (2000: 197), las personas socialmente inteligentes y con un alto grado de empatía conectan más fácilmente con los demás, tengan mayores habilidades para comunicarse en lengua extranjera.

Otro aspecto que los investigadores han tenido en cuenta, al haber encontrado diferencias bastante significativas en el aprendizaje de un idioma, es la actitud y su relación con el género, la motivación y el rendimiento. En términos generales se conceptualiza la actitud como la tendencia a actuar con una determinada intensidad hacia algún objetivo determinado. En este supuesto se encuentran dos características de la actitud que suelen ser constantes en todas las definiciones consultadas. La primera alude a la tendencia a actuar, disposición de 
actuación, que parece ir asociada con una orientación y predisposición del individuo, favorable o desfavorable, positiva o negativa, a operar hacia determinados aspectos del entorno, que en nuestro caso sería el aprendizaje de la lengua meta. El segundo aspecto es la intensidad con la que un individuo está dispuesto actuar; esta tendencia a la respuesta puede ser más o menos intensa dependiendo de cada individuo y de los diferentes aspectos del entorno de la comunidad lingüística-cultural de la lengua meta. Krech, Crutchfield y Ballachey (1962) añaden un tercer rasgo, la permanencia relativa y matizan que las actitudes son "sistemas duraderos de evaluaciones positivas o negativas, sentimientos emocionales y tendencias a la acción favorable o contraria respecto de unos objetos sociales" (1962: 139).

En el contexto de aprendizaje de un idioma la actitud se caracteriza, en general, como una reacción evaluativa basada en las opiniones y creencias del individuo hacia un determinado referente. Según Burstall (1975) las mujeres muestran una actitud más favorable que los hombres ante la lengua meta y logran mejores resultados dentro del contexto académico. El autor fundamenta que el mercado laboral exige más el conocimiento de idiomas a las mujeres que a los hombres. Anteriormente, en 1971, los trabajos de Robinson concluyeron con los mismos resultados y atribuyeron estas diferencias de actitud entre ambos al hecho de que ser buen alumno en una lengua extranjera era considerado poco masculino. Las investigaciones realizadas por Prada (1993) en las que valora parámetros como la nota escolar, la motivación instrumental e integradora, la actitud hacia los ingleses y americanos, etc., concluyen que la actitud y la motivación del grupo de mujeres es más positiva que la de los hombres. Otros estudios (Prada y Fernández, 1998) mostraron nuevamente que los hombres presentan actitudes menos positivas que las mujeres, tanto respecto a los hablantes y cultura de la lengua meta como al propio proceso de enseñanza-aprendizaje.

Por consiguiente, no existe un claro consenso entre la influencia de los factores motivacionales-emocionales y el género. En nuestra investigación queremos inducir al profesorado a reflexionar y a que nos relate, desde la perspectiva de su actividad docente, si estos factores, que directa o indirectamente, positiva o negativamente, intervienen en el proceso de aprendizaje de idiomas, influyen de forma diferente en las alumnas que en los alumnos. Los resultados pueden ayudar a determinar si existe relación entre el género y dichos factores además, si se detectan diferencias de género, a estimar si debemos promover cambios en la metodología didáctica.

\section{Metodología}

Para conocer las opiniones de los docentes realizamos una encuesta con preguntas de respuesta abierta, a modo de relato biográfico. Para la interpretación y el análisis del material contamos, siguiendo a Bolívar et al. (2001: 147), con cuatro elementos: un narrador, que cuenta sus vivencias; un intérprete o investigador, que elabora el informe de los relatos; textos donde se recoge lo que es narrado y por último lectores a los que se dirige el informe. Además, partimos de un marco teórico como base conceptual para la interpretación de los registros o textos; la reflexión del acto de lectura, de interpretación y de extraer conclusiones (Lieblich et al., 1998). Este tipo de investigación narrativa tiene la ventaja de respetar la voz o el discurso de los profesores por lo que la tarea interpretativa también se relega al lector. 


\subsection{Elaboración, diseño y validación del cuestionario}

Optamos por preguntas sin respuestas preestablecidas, pues permiten conocer la opinión de cada sujeto, expresada libremente y contada con sus propias palabras, sin límite de espacio para responder, permitiendo añadir observaciones y comentarios. Consideramos que con este diseño se obtener una información amplia, generosa y con múltiples matices.

Para la validación del cuestionario realizamos un "Pretest" y lo aplicamos a 12 profesores expertos, escogidos de tal forma que representaran la diversidad de sujetos a los que iba a ser dirigido, procedentes de las etapas educativas de Primaria, Secundaria y Universidad con los siguientes objetivos: evaluar la compresión, fiabilidad y validez de cada una de las preguntas.

\subsection{Definición y selección de la muestra a encuestar}

Nos interesa conocer los pensamientos de docentes en los tres niveles educativos existentes en nuestro entorno, por lo que seleccionamos una muestra de profesores de Primaria, Secundaria y Universidad que impartían los tres idiomas más habituales en nuestro entorno, inglés, francés y alemán. El interés de nuestra investigación no reside en el número de sujetos encuestados sino en la profundidad de sus observaciones, por ello, consideramos que la muestra de encuestados debería poseer un perfil determinado para responder consecuentemente al cuestionario. Uno de los criterios que tuvimos en cuenta para la selección de los informantes fue que se encontraran inmersos en preocupaciones específicas relacionadas con los procesos de enseñanza-aprendizaje de idiomas, y que a lo largo de su carrera profesional se hubieran interesado por la didáctica de idiomas. Sus aportaciones podrían enriquecer este entramado tan complejo de la influencia de motivación y los sentimientos en el aprendizaje de lenguas extranjeras en los alumnos y las alumnas.

\subsection{Metodología para la entrega y recogida del cuestionario}

Se distribuye el cuestionario a los profesores de manera personalizada. Ello permitió explicar detalladamente el objeto de la encuesta y la finalidad del estudio. Enviamos la encuesta a noventa docentes y recibimos la respuesta (anónima) de cincuenta y ocho. No se estableció ningún criterio de selección en cuanto al perfil de los profesores en relación al género, idioma que impartían, nivel educativo y años de experiencia, aunque son factores que tendremos en cuenta para el análisis de los datos.

\subsection{Procesamiento de los datos}

Para el manejo y procesamiento de la ingente cantidad de información y su interrelación con los diferentes encuestados se empleó un programa informático Atlas/ti5 versión 3.03 (Muñoz Justicia, 2006) que facilitó la organización y segmentación de los textos en pasajes o citas, codificarlos y escribir comentarios y anotaciones. El procesamiento de los datos requiere una codificación a partir de la cual se establecen una serie de categorías que se van afinando y modificando sucesivamente a medida que se avanza en el análisis. De esta manera se establece una interacción continua entre la recogida de datos de las encuestas y 
el análisis que se va realizando. Dicho proceso se realiza en varias fases: lectura inicial de los datos, codificación y categorización y obtención de conclusiones (LeCompte, 2000). Este proceso se retroalimenta a sí mismo pues supone una constante revisión, relectura de las encuestas, adecuación de los códigos a medida que se progresa en su estudio y valoración. Previamente al análisis de los cuestionarios, realizamos un exhaustivo trabajo de categorización y recategorización de las respuestas. Este programa proporciona, además, datos cuantitativos, sin embargo el análisis desde esta perspectiva se realizó con la intención de enriquecer nuestra investigación, puesto que nuestro estudio se ha efectuado desde una perspectiva fundamentalmente cualitativa. Para el análisis de los textos se estableció un sistema de codificación que consistió en identificar cada encuesta con una letra (P) seguida de un número $(\mathrm{P} 1, \mathrm{P} 2, \ldots)$, sistema que utilizaremos en las citas en este artículo.

\section{AnÁlisis de la encuesta y PResentación de los Resultados}

En general, ¿Cree usted que existe diferencia de género en el papel que juega la motivación en el proceso de enseñanza-aprendizaje y de manera especial, en el aprendizaje de una lengua extranjera?

Un número elevado de profesores no ha observado diferencias de género en el papel que desempeña la motivación:

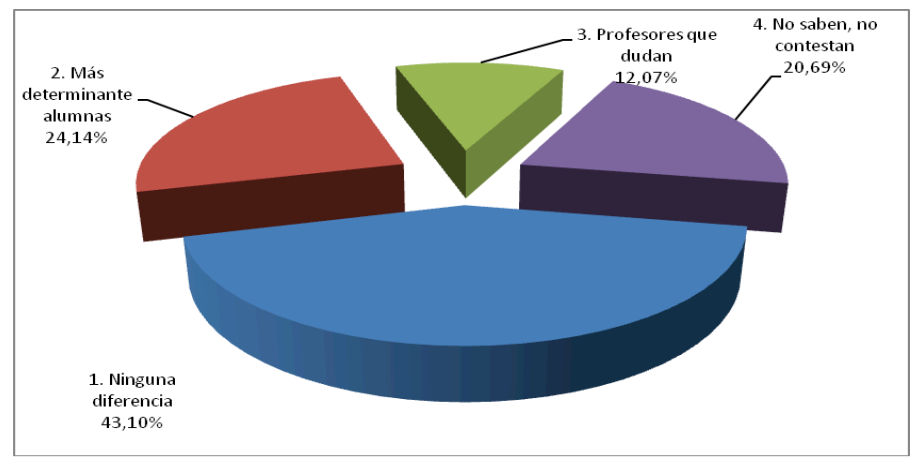

Figura 1. Distribución de respuestas por categorías

\subsection{Análisis pormenorizado de cada categoría}

\section{- No existen diferencias de género}

Aproximadamente la mitad de los encuestados (25) afirma que no existen diferencias significativas entre ambos géneros respecto al papel que desempeña la motivación en el aprendizaje, y de manera concreta en relación al idioma. La mayoría responde de manera clara y concisa: "No creo haber percibido nunca diferencias entre alumnas y alumnos" (P37).

Algunos encuestados explican que la motivación no depende del género sino del mismo sujeto y de las influencias que éste recibe del contexto de aprendizaje: "No veo en este 
sentido otro horizonte que no sea el de un individuo (con sus motivaciones personales) en el seno de un grupo (que recibe unas motivaciones directas o indirectas)" (P3). Coincidiendo con esta opinión, un profesor de Universidad y otro de Primaria añaden que las diferencias radican en la naturaleza de la motivación, en las disparidades respecto a las materias de interés de los estudiantes: "Creo que el sexo no interviene en el grado de motivación, aunque sí puede ser cierto que a ellas les motiva la moda, el cine, la música y a los chicos les llaman la atención las noticias de páginas deportivas y de juegos." (P10).

Sin embargo, ciertos docentes, aunque aseveran que la motivación es independiente del género, matizan que ellas perseveran mas en el estudio, obtienen mejores resultados y se implican de manera más responsable en la realización de las tareas: "La motivación en las alumnas funciona igual que en los alumnos, pero el resultado es mejor en las alumnas porque ellas aceptan mejor la parte de esfuerzo y trabajo que tiene toda asignatura" (P1). Otros informantes afirman que la diferencia de género se encuentra en la manifestación de las emociones pero no en la motivación: "No tengo experiencias que me permitan concluir que la motivación está ligada a la diferencia de sexos. La manifestación de emociones, quizá sí sea diferente "(P35). Un argumento común a este grupo de informantes es que las alumnas maduran antes que los alumnos, un factor que influye favorablemente en el aprendizaje.

Un docente, que aunque de igual forma desvincula el papel de la motivación del género, atribuye a las mujeres mayores habilidades para el aprendizaje de idiomas: "El papel es el mismo, aunque por norma general las niñas tienen más facilidad que los niños para el aprendizaje de idiomas" (P14). Estas consideraciones, como se puede observar en la siguiente categoría, son la causa de que algunos enseñantes afirmen que la motivación desempeña un papel más determinante en las alumnas.

\section{- La motivación juega un papel más determinante en las alumnas}

En esta categoría se advierten argumentos ya comentados en la anterior, sin embargo, éstos sirven para justificar que la motivación, en opinión de 14 profesores, desempeña un papel más determinante en las alumnas. Como ejemplo presentamos el texto de un docente del ámbito educativo de Secundaria:

Las alumnas maduran antes, expresan mejor sus emociones y tienen mayores facilidades para el aprendizaje, prestan más atención en clase, son más receptivas, más responsables, tienen mejor predisposición hacia el aprendizaje, se esfuerzan más por adquirir mejores notas, son más tranquilas y cuidadosas con sus trabajos, y más conscientes de la necesidad de aprender en general (P13).

Estos argumentos los esgrimen sobre todo enseñantes que proceden de los ámbitos educativos de Primaria y Secundaria. Un profesor de Primaria de francés expresa: "En general las chicas son "mejores alumnas": más atentas en clase, más voluntariosas, más constantes [...] más adaptadas al sistema escolar" (P17). Una profesora de alemán de Secundaria (P56) manifiesta que ellas, por lo general, se encuentran motivadas, mientras que los alumnos sólo se motivan cuando tienen perspectivas de realizar estudios universitarios:

En mi escuela las alumnas muestran una motivación mucho mayor hacia los estudios en general, y ante la lengua extranjera, en particular. Salvo un número reducido de 
chicos, claramente encaminados hacia la universidad, el resto considera que no es necesario saber un idioma para ponerse a trabajar cuanto antes (P56).

Otro docente de Secundaria de inglés (P41) basa sus afirmaciones en que más del $80 \%$ del profesorado joven de idiomas son mujeres. Además, atribuye a las alumnas características especiales que benefician el proceso de aprendizaje:

En general, creo que las mujeres tienen mejor predisposición para los idiomas y menos inhibición, menos sentimientos de ridículo y ansiedad frente a problemas como el entrenamiento fonético, por ejemplo. También influye el hecho genérico de que en estas edades escolares, las chicas son algo más maduras que los chavales de su misma edad (P41).

\section{- Profesores que dudan}

Siete enseñantes dudan de la relación entre la motivación y el género, sin embargo, afirman la existencia de diferencias significativas entre la actitud de las alumnas y la de los alumnos frente al aprendizaje. Otorgan al primer colectivo mayor responsabilidad, predisposición y preocupación por la aprehensión de un idioma y mayor interés hacia otras materias. No obstante, atribuyen estas diferencias o a factores como los socio-culturales:

En general, los resultados son más altos en las alumnas y también muestran un mayor interés en las clases. Quizás esto se debe a la motivación o a una cuestión cultural que rige el comportamiento social de las chicas y los chicos (normalmente ellas más estudiosas y responsables que ellos). Las alumnas son más metódicas, más constantes en el estudio y, por lo tanto, se enfrentan a la lengua extranjera con mayor probabilidad de éxito, frente a ellos, que tienden a estudiar a última hora y por lo tanto, sus conocimientos (en el caso del idioma) no suelen perdurar. (P48).

Algunos profesores manifiestan que el número de alumnas que cursan una segunda lengua supera al de alumnos, por ello concluyen que la motivación desempeña un papel más importante en las aprendices. Sin embargo, los informantes muestran sus dudas puesto que "[...] las causas para la elección del segundo idioma pueden ser múltiples o bien circunstanciales" (P28). Un docente concluye su relato ensalzando las habilidades y actitudes de las alumnas: "No lo sé. Sólo puedo decir que las chicas eligen más el segundo idioma, que son mejores en los estudios que los chicos, que trabajan más y que son más maduras" (P46). Algunos encuestados, además, inciden en la facilidad de las alumnas para comunicarse: "Mi experiencia docente me dice que las chicas son más receptivas a todo tipo de actividades y se prestan más a entablar conversación sobre cualquier tema, y esto en el aprendizaje de cualquier idioma es muy positivo, pero en absoluto ajeno a otras disciplinas (P8).

Este colectivo de profesores, aunque sus respuestas se pueden interpretar como más favorables hacia el grupo de alumnas, expresan sus dudas puesto que esas diferencias se generan en el entorno familiar:

Los padres fomentan en las niñas el gusto por el idioma" (P25). 
Las madres se preocupan más por el aprendizaje de idiomas y esa diferencia la ven las hijas, quizás sea por imitación, o cercanía con la madre" (P8).

A continuación analizamos los resultados de la cuestión que hace referencia a las influencias de las emociones:

3.2. En general, ¿cree usted que las emociones juegan el mismo papel en las alumnas que en los alumnos y, de manera especial, en relación con el aprendizaje de una lengua extranjera?

La opinión mayoritaria es que no existen diferencias de género en el papel que desempeñan las emociones en el aprendizaje de un idioma. En la figura 2 presentamos gráficamente estos datos:

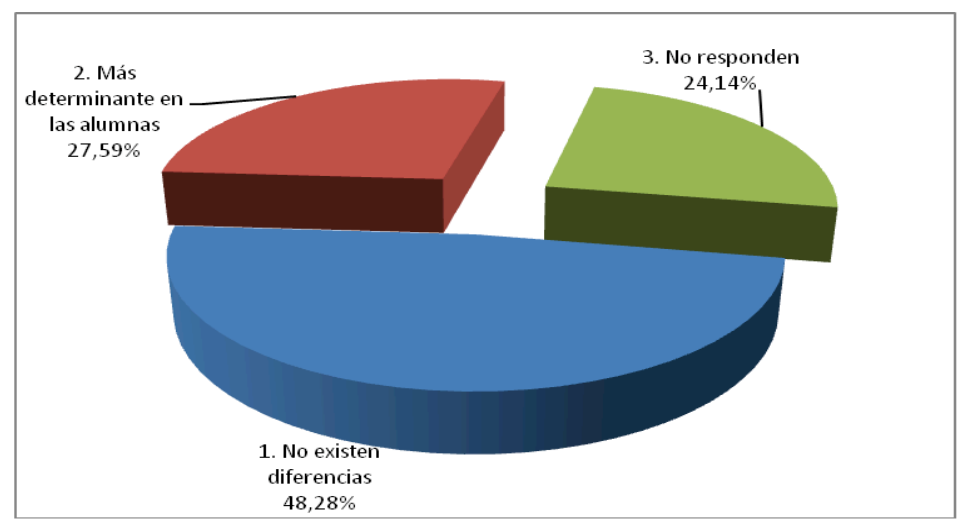

Figura 2. Distribución de respuestas por categorías

\subsubsection{Análisis pormenorizado de cada categoría y subcategoría}

\section{- No existen diferencias}

Un número elevado de profesores (28) manifiesta, de manera concluyente y sin apenas justificar sus respuestas que "no existen diferencias" (P9). Opinan que los afectos dependen de las circunstancias y de los intereses individuales. Si existen diferencias se tendrían que explicar "[...] en términos de individuo y no de sexo" (P3). Los informantes relatan que en la generación de emociones influyen múltiples factores fundamentalmente las experiencias educativas anteriores o el contexto familiar - "No creo que sea tanto una cuestión de género como de talante, de predisposición innata o inculcada desde anteriores niveles formativos, o desde la familia" (P39) —. Para un encuestado "las emociones están sujetas a la implicación del alumno en las actividades de aula [...] serán mayor en tanto en cuanto el profesor logre tocar esos intereses" (P10). Un enseñante de Primaria otorga a las alumnas en la adolescencia mayor capacidad para expresar sus sentimientos: "No especialmente. Creo que las alumnas, 
en estas etapas de la adolescencia suelen dejarse llevar mucho más por las emociones que los alumnos, pero en general su proceso de aprendizaje suele ser bastante similar" (P42).

\section{- Las emociones juegan un papel más determinante en las alumnas}

Dieciséis profesores afirman que las emociones desempeñan un papel más determinante en las alumnas. Los argumentos aducidos son los siguientes:

\section{- Las alumnas son más espontáneas, sensibles, emotivas}

Diez docentes basan sus reflexiones en las diferencias observadas respecto a la manifestación de las emociones. Las alumnas son más espontáneas, expresan su agrado o desagrado ante el aprendizaje de idiomas de manera más emotiva y natural que sus compañeros “ $\ldots$ lo que podría hacer pensar que manifiestan más sus afectos o desafectos con el aprendizaje de la lengua extranjera" (P37). Por el contrario, los chicos reservan sus sentimientos o muestran sus emociones de acuerdo con los patrones sociales establecidos: "Mientras que los alumnos muchas veces se ven coartados por las mismas (las alumnas). Los chicos deben mostrar una apariencia ante los demás $\mathrm{y}$, en ocasiones, esa apariencia va reñida con la expresión de sus emociones" (P56).

Así mismo destacan que las alumnas son más receptivas, responsables, ordenadas; características que contribuyen, como apunta $\mathrm{P} 7$, a solventar dificultades personales como la vergüenza, el miedo al ridículo o la frustración. Estos docentes opinan que ellas se caracterizan por su mayor responsabilidad y amor propio lo cual contribuye a superar las emociones negativas "[...] a pesar de tener, a mi parecer, más sentido del ridículo que los chicos, se esfuerzan más por superarlo a la hora de participar en clase" (P7). Otro profesor añade: "... ellas son más abiertas y receptivas a las tareas novedosas, pues se implican más que ellos" (P54). Un encuestado explica que estas diferencias emocionales no sólo se observan en la clase de idiomas: "(...) las emociones juegan papel más determinante en alumnas pero no solamente en relación con las lenguas extranjeras" (P2).

Otro profesor apunta que la comisión de errores genera menos problemas en las alumnas que en los alumnos: "A ellas: les cuesta menos expresar lo que sienten y no les importa tanto cometer errores a la hora de expresarse en otro idioma" (P8).

Finalmente, cinco profesoras de Primaria explican que “(...) en la mayoría de los casos ellas son más sensibles para captar las necesidades y empatizar” (P47). En esta línea argumental P15 manifiesta que los alumnos con un alto grado de empatía logran mejores éxitos y se enfrentan al aprendizaje con mayor entusiasmo debido a su capacidad de entender mejor la otra cultura. Tres docentes de Primaria sostienen que en los niños se observan mayores prejuicios que en las niñas según avanza su proceso de madurez: "En edades tempranas ambos sexos expresan sus emociones de forma espontánea, cuando van creciendo, los niños sienten más prejuicios, supongo que por presiones sociales. Creo que este hecho influye positivamente en ellas y negativamente en ellos" (P25). Esa diferencia se comienza a notar a partir de una edad: "A determinadas edades creo que sí. En E. Primaria, de 10 a 12 años, es donde más lo noto" (P12).

\section{- Motivos socio-culturales}

Las diferencias de género se observan en la expresión de las emociones cuyas causas son culturales-educacionales: 
[...] puede que se deba a razones culturales, a la manera en la que cada sociedad construye la feminidad y la masculinidad normativas. A las niñas se las suele educar para comportarse e incluso sentir de una forma diferente que a los niños, y también perciben los estudios de manera diferente, les dan más importancia y son más aplicadas, por lo general. Suelen tener más fuerza de voluntad y ser menos tímidas (porque también estudian más por lo general y por lo tanto sus competencias en el idioma son mayores). Pero creo que no es una cuestión fisiológica sino cultural (P48).

otro encuestado expresa: “[...] a las mujeres se las estimula más desde que nacen y se les enseña otro tipo de comportamientos y por esta educación diferenciada, los chicos comunican sus emociones más torpemente" (P6).

\section{Discusión de los resultados}

Un número elevado de encuestados aprecia diferencias entre la actitud de unas y de otros hacia el aprendizaje. Los resultados de la encuesta coinciden con las investigaciones de Prada (1993), Prada y Fernández (1998), en las que se concluye que tanto la actitud como la motivación del grupo de mujeres resultan ser más positivas que la de los varones. Los encuestados las describen como un colectivo claramente diferenciado, con mayores habilidades, elevada responsabilidad por la ejecución de las tareas, más metódicas, más comprometidas con el proceso de enseñanza-aprendizaje, más trabajadoras, obtienen mejores resultados, presentan mayor predisposición hacia el aprendizaje y son más curiosas que los alumnos. Estos términos están relacionados con el concepto de motivación intrínseca, que surge por el simple deseo de realizar aquello que despierta interés y genera satisfacción (Williams y Burden, 1999). Las investigaciones llevadas a cabo por Stewart-Strobelt y Huabin (2003: 162) acerca de las causas por las que los estudiantes eligen una lengua extranjera concluyen que las alumnas poseen una elevada motivación integradora en comparación con los alumnos ( $70 \%$ y $50 \%$ respectivamente).

Los profesores de Primaria y Secundaria relacionan la motivación con el grado de madurez del individuo y argumentan que las alumnas maduran antes lo cual explica su mayor motivación. La madurez dirige al aprendiz a solucionar determinadas necesidades como finalizar antes los estudios, conseguir mejores calificaciones, buscar un trabajo... Otal et al. (1992) concluyeron que la necesidad de aprender un idioma favorece la obtención de resultados positivos en el aprendizaje, aunque la actitud hacia los hablantes de la otra cultura sea negativa.

Para algunos informantes, otro factor indicativo es la existencia de un número más elevado de mujeres en la enseñanza. Además de estos datos, se da la circunstancia de no haber encontrado ningún texto en el que se abogue por un mayor influjo de la motivación en los alumnos.

En relación a la ascendencia de las emociones observamos que los profesores emplean argumentos muy similares a los desarrollados en relación a la influencia de la motivación. Además, resulta llamativo que ningún informante se pronuncia a favor de que las emociones desempeñen un papel más determinante en los varones. De nuevo, las describen como 
sujetos con actitudes y sentimientos más positivos hacia el aprendizaje, son más sensibles, comunicativas, expresivas, abiertas, espontáneas, con menos sentido del ridículo, atributos que facilitan el éxito en el aprendizaje. Su naturaleza más comunicativa y expresiva la atribuyen a razones educativas y culturales.

Compartimos con Tannen (1994) el no atribuir juicios de valor a estas diferencias descritas, puesto que las interpretamos como diferencias culturales en el discurso de hombres y mujeres, debidas en gran parte a su desigual socialización a través del lenguaje. Esta autora defiende que las mujeres se implican de manera más personal en las tareas y asocia al discurso femenino la diplomacia al expresar desacuerdos para evitar incomodidad al interlocutor.

Marcos-Llinás (2007) avala que las mujeres tienen más facilidad que los hombres para el aprendizaje de idiomas y fundamenta sus conclusiones en la curiosidad innata en la naturaleza comunicativa y expresiva. Nuestro estudio, sin embargo no acredita que la curiosidad y la naturaleza comunicativa de las alumnas sean de origen innato sino social, especialmente familiar, pero coincide con la autora en que las mujeres, en edades tempranas, poseen actitudes más positivas y una motivación más alta que los hombres.

En relación a las emociones la opinión mayoritaria es que éstas desempeñan un papel más determinante en las mujeres. Varios encuestados destacan que las alumnas poseen mayor empatía, resultados que coinciden con los estudios llevados a cabo por Goleman: "Existen cientos de estudios que han puesto de manifiesto que las mujeres suelen ser más empáticas que los hombres, al menos en lo que se refiere a su capacidad para captar los sentimientos que se reflejan en el rostro, el tono de voz y otro tipo de mensajes no verbales (2000: 214)". En general, la empatía se define como "el proceso de ponerse en la piel de otra persona" (Arnold y Brown 2000: 36). Los encuestados sostienen que esta identificación mental y afectiva de uno respecto al estado de ánimo de otro, la capacidad para entender y escuchar al compañero, al grupo o a personas de otra cultura favorece la aprehensión de la otra lengua-cultura. Diversas investigaciones han evidenciado que este sentimiento dirige la atención hacia la competencia intercultural y que, como afirma Trujillo Sáez (2005), fomenta la conciencia colectiva hacia la diversidad, el respeto hacia otras formas de pensamiento de enseñar y aprender.

\section{Conclusiones Y REFleXión Final}

En virtud de las respuestas podemos concluir que ningún enseñante declara que la motivación y emoción desempeñan un papel más determinante en los varones.

La mayoría reconoce que las alumnas poseen características especiales que favorecen la aprehensión de un idioma:

- Predisposición a adquirir conocimientos nuevos

- Más participativas y comprometidas con el aprendizaje

- De naturaleza más expresiva, les gusta hablar, opinar

- Actitud más positiva hacia el aprendizaje

- Curiosidad por conocer nuevas cosas

- Mayores habilidades

- Más responsables

- Implicación en las tareas 
- Metódicas

- Comprometidas con el aprendizaje

- Perseverantes en el estudio

- Mayor madurez

En relación con las emociones podemos definir ciertos rasgos, más propios de las alumnas, que contribuyen a paliar los afectos negativos y a generar, fomentar y mantener las emociones positivas hacia el aprendizaje:

- La empatía

- Capacidad para expresar las emociones

- Mayor sensibilidad y emotividad

- Capacidad de recepción

- Habilidades para solventar con mayor facilidad momentos de estrés y ansiedad

- Mayor desinhibición

Los informantes aclaran que las diferencias de género se deben a motivos socio-culturales (especialmente la influencia del profesor y de la familia) y de madurez del alumno no a causas de origen innato.

Los argumentos esgrimidos por los profesores de Primaria y Secundaria son congruentes con los expresados por los docentes en la Universidad. Los primeros aducen que en edades tempranas la motivación y la emoción ejercen más influencia en las alumnas puesto que maduran antes mientras que, como han observado los profesores de Universidad, cuando el alumno alcanza su madurez cognitiva no hay diferencias de género.

Así mismo, la investigación ha proporcionado datos sobre los efectos de determinadas emociones como el estrés, la tensión, el sentimiento de ridículo o la ansiedad. Las opiniones más generalizadas son que en edades tempranas se eliminan con mayor facilidad estos filtros afectivos que impiden o dificultan la adquisición de un idioma. Incluso algunos relatores comparten la opinión de que las alumnas, en edades tempranas aprenden con mayor facilidad pues actúan espontáneamente y sin ansiedad.

Respecto al idioma que imparten, no encontramos diferencias de opinión entre los docentes de Alemán, Francés e Inglés. Sin embargo, se observan discrepancias respecto al género, ámbito educativo y años de docencia. Tanto las mujeres como los varones estiman mayoritariamente que no existen diferencias de género en el papel que juegan la motivación y las emociones, aunque es de destacar la existencia de un número mayor de varones que comparte esta opinión.

En relación al ámbito educativo existen algunas divergencias. Los profesores que imparten docencia en la Universidad no han observado diferencias de género en la influencia que ejercen la motivación y la emoción, por el contrario un número elevado de docentes de Primaria y Secundaria afirman que estas son más determinante en las alumnas.

En cuanto a los años de docencia observamos que todos los profesores con más de veinte años de experiencia profesional manifiestan que la motivación y las emociones ejercen el mismo papel en las mujeres que en los varones, sin embargo no todos los profesores noveles comparten esa opinión y caracterizan a las alumnas como más extrovertidas, receptivas, emotivas.

Para finalizar, proponemos crear en el contexto de aprendizaje, un entorno libre de presiones y elaborar actividades, consensuadas con el alumno, de carácter lúdico que 
incrementen el interés, la motivación y que no comprometan emocionalmente de manera negativa a los participantes. La investigación ha puesto de manifiesto la fascinante y compleja labor del profesor como guía del proceso de enseñanza-aprendizaje. Sobre él recaen serias responsabilidades: influye en los procesos de pensamiento, facilita la comprensión, vela para que los estudiantes no olviden lo aprendido, proporciona nuevos conocimientos, se le encomienda la tarea de mantener o aumentar la motivación además de activar o despertar motivos para aprender. A todo lo anterior hay que añadir que el docente debe tener en cuenta la distribución grupal de los alumnos, de tal forma que alumnas y alumnos se influyan de manera positiva, pues de ello depende un adecuado desarrollo de los aspectos socio-emocionales y comunicativos.

Por todo ello, abogamos por una sólida formación psicopedagógica y didáctica de los profesores que promueva la adquisición de conocimientos y destrezas que permitan el manejo eficaz de las emociones en el aula de idiomas, que ofrezca un conocimiento empático para que, desde el vínculo didáctico que se establece con los alumnos, el docente sea capaz de reconocer y modificar comportamientos.

\section{REFERENCIAS BIBLIOGRÁFICAS}

Alonso Tapia, J. (2005). Motivar en la escuela, motivar en la familia. Madrid: Morata.

Arnold, J. (2000). La dimensión afectiva en el aprendizaje de idiomas. Madrid: Cambridge University Press.

Arnold J. y Brown, D. (2000). "Mapa del Terreno", en Arnold J. La dimensión afectiva en el aprendizaje de idiomas. Madrid: Cambridge University Press, 19-41.

Bernaus, M. (ed.) (2001). Didáctica de las lenguas extranjeras en la educación Segundaria Obligatoria. Madrid: Síntesis educación.

Bolívar, A., Domingo, J. and Fernández M. (2001). La investigación biográfico-narrativa en educación. Enfoque y metodología. Madrid: La Muralla.

Burstall, C. (1975). "Primary French in Ihe balance", in Educalional Research, 17, 193-198.

Campbell, C. M. (1999). "Language anxiety in men and women: Dealing with gender difference in the language classroom", in YOUNG, D. J. (Ed.), Affect in foreign language and second language learning: a practical guide to creating a low-anxiety classroom atmosphere. Boston: McGraw Hill, 19-41.

Campbell, C. M. y Shaw, V. (1994). "Language anxiety and gender differences in adult second language learners: Exploring the relationship", in Klee, C. (ed.), Faces in a crowd: The individual learner in multisection courses. Boston: Heinle \& Heinle.

Dörnyei, Z. (2009). "Individual Differences: Interplay of Learner Characteristics and Learning Environment", in Language Learning, 59: 230-248.

Entwistle, N. J. and Wilson, J. D. (1977). Degrees of Excellence: the Academic Achievement Game. Londres: Hodder and stoughton.

Eysenck, H. J. and Wilson G. D. (1981). Psicología del sexo. Barcelona: Herder.

Gardner, R. C. and Lambert W. E. (1972). Attitudes and Motivation in Second-Language Learning. Rowley: Newbury House Publishers.

Goleman, D. (2000). Inteligencia emocional. Barcelona: Kairós.

Guirora, A., Brannon, R. and Dull, C. (1972). "Empathy and Second Language Learning", in Language Learning 22, 111-130. 
Krashen, S. (1985). The Input Hypothesis: Issues and Implications. Nueva York: Longman.

Krech, D., Crutchfield, R. S., and Ballachey, E. L. (1962). Individual in society. A Textbook of Social Psychology. New York: McGraw-Hill Book Company.

Ledoux, J. (1999). El cerebro emocional. Barcelona: Planeta.

Lecompte, M. (2000): "Analyzing Qualitative data", in Theory Into Practice, 39 (3), 146-154.

Lieblich A., Tuval-Mashiach, R. and Zilbert, T. (1998). Narrative research. Reading, analysis, and interpretation Thousand Oaks, CA: Sage.

Marcos-Llinás, M. (2007). "Variables afectivas en la clase de lenguas extranjeras", en Interlingüistica, 17, 676-678.

Miller, C.D., Finkey, J., and Mckinley, D.L. (1990). "Learning approaches and motives: male and female differences and implications for learning assistance programs", in Journal of College Student Development, 31(2), 147-154.

Muñoz, C. (2002). Aprender idiomas. Barcelona: Paidós.

Muñoz Justicia, J. (2005). Análisis cualitativo de datos textuales con Atlas.ti 5, versión 3.03 Disponible en http://www.fcp.uncu.edu.ar/upload/Atlas5_manual.pdf. [Consulta: 17 de abril de 2006].

Otal, J. L., Artigas, A and Alargón, J. (1992). "Análisis de la motivación y técnicas de aprendizaje de inglés de los estudiantes de enseñanza media de Zaragoza”, en Actas del VIII Congreso Nacional de Lingüistica Aplicada, 545-570.

Oxford, R. and Sherin, J. (1994). "Language Learning Motivation: Expanding the Theoretical Framework", in The Modern Language Journal, 78, 12-28.

Pavlenko, A. (2002). "Bilingualism and Emotions", in Multilingua, 21, 45-78.

Prada, E. De and Fernández, M. V. (1998). "Actitud, motivación y percepción del aprendizaje según el sexo en el estudio del inglés con multimedia", en Mayobre P. y Caruncho, C. (Coord.), Novos dereitos: Igualdade, diversidade e disidencia. Santiago: Tórculo Artes Gráficas, 275-290.

Prada, E. De. (1993). Aspectos psicolingüisticos del aprendizaje de una lengua extranjera. Universidad de Santiago de Compostela: Servicio de Publicaciones e Intercambio Científico.

Ray Crozier, W. (2001). Diferencias individuales en el aprendizaje. Personalidad y rendimiento escolar. Madrid: Nancea.

Rubio Alcalá, F. D. (2004). La ansiedad en el aprendizaje de idiomas. Huelva: Universidad de Huelva.

Solsky, B. (1989). Sociolinguistics. Oxford: Oxford University Press.

Stewart-Strobelt, J. and Huabin CH. (2003). "Motivations and Attitudes Affecting High School Students' Choice of Foreign Language", in Adolescente, 38 (149), 161-170.

Tannen, D. (1994). Gender and Discourse. New York: Oxford University Press.

Trujillo Sáez, F. (2005). "Entorno a la interculturalidad: reflexiones sobre cultura y comunicación para la didáctica de la lengua", en Porta Linguarum, 4, 23-39.

Wazel, G. (ed.) (2001). Interkulturelle Kommunikation in Wirtschaft und Fremdsprachenunterricht. Frankfurt am Main: Peter Lang.

Williams, M. and Burden, R. L. (1999). Psicología para profesores de idiomas. Enfoque del constructivismo social. Madrid: Cambridge University Press. 